PRAXIS

ecucativa

Universidad Nacional de La Pampa

Facultad de Ciencias Humanas

Instituto de Ciencias de la Educación

para la investigación interdisciplinaria

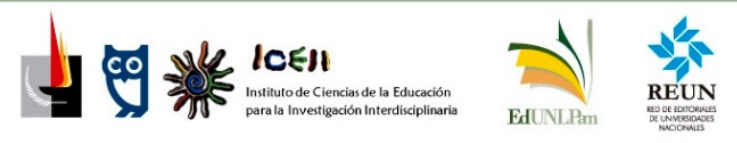

ISSN 2313-934X

SANTA ROSA, LA PAMPA, ARGENTINA

Correo electrónico: iceii@humanas.unlpam.edu.ar

Disponible en https://cerac.unlpam.edu.ar/index.php/praxis

Lo escolar a través de la pantalla. Trabajo docente en Chiapas, México. Artículo de Germán Alejandro García Lara, Soledad Hernández Solís, Irma Hernández Solís, Oscar Cruz Pérez, Jesús Ocaña Zúñiga y Carlos Eduardo Pérez jiménez. Praxis educativa, Vol. 26, No 1 enero abril 2022. E - ISSN 2313-934X. pp. 1-23. https://dx.doi.org/10.19137/praxiseducativa-2022-260103

Esta obra se publica bajo Licencia Creative Commons 4.0 Internacional CC BY- NC-SA Atribución, No Comercial, Compartir igual

\title{
Lo escolar a través de la pantalla. Trabajo docente en Chiapas, México
}

School work through the screen. Teaching work in Chiapas, Mexico

Escola através da ecrã. Trabalho docente em Chiapas, México

\section{Germán Alejandro García Lara}

Universidad de Ciencias y Artes de Chiapas, México

ORCID 0000-0002-7736-9717

german.garcia@unicach.mx

\section{Soledad Hernández Solís}

Universidad de Ciencias y Artes de Chiapas, México

ORCID 0000-0001-5621-986X

soledad.hernandez@unicach.mx

\section{Irma Hernández Solís}

Universidad de Ciencias y Artes de Chiapas, México

ORCID 0000-0001-7335-7519

irma.hernandez@unicach.mx

\section{Oscar Cruz Pérez}

Universidad de Ciencias y Artes de Chiapas, México

ORCID 0000-0003-2452-2834

oscar.cruz@unicach.mx 


\section{Jesús Ocaña Zúñiga}

Universidad de Ciencias y Artes de Chiapas, México

ORCID 0000-0003-0300-0797

jesus.ocana@unicach.mx

\section{Carlos Eduardo Pérez Jiménez}

Universidad de Ciencias y Artes de Chiapas, México

ORCID 0000-0001-7051-9017

carlos.perez@unicach.mx

Recibido: 2021-09-05 | Revisado: 2021-11-09 | Aceptado: 2021-11-17

\section{Resumen}

El estudio analiza el trabajo escolar de docentes de escuelas urbanas y rurales, de preescolar y primaria, en Chiapas, ante la emergencia sanitaria por COVID-19. La investigación es de tipo cualitativa hermenéutica, llevada a cabo a partir de entrevistas a 22 profesores/as. El análisis posibilitó la construcción de tres bloques temáticos: A través de la pantalla. Penurias, angustias y malestares, El trabajo escolar. Contextos públicos y privados de la enseñanza y escuelas en resistencia; y Programa Aprende en casa. Actualizar, en la memoria, las formas de acción pedagógica y ético-políticas con que el profesorado ha hecho frente a esta inédita condición socioeducativa ofrece un marco para repensar la reconfiguración de la relación entre los agentes escolares y las familias, para pugnar por una mayor equidad del sistema educativo.

Palabras clave: profesorado; educación básica; pandemia; trabajo escolar

\section{Abstract}

The study analyzes the school work of teachers from urban and rural preschool and primary schools in Chiapas, in response to the health emergency caused by Covid-19. The research is of a qualitative hermeneutic type, carried out from interviews with 22 professors. The analysis enabled the construction of three thematic blocks: Through the screen. Hardship, anguish and discomfort, School work. Public and private contexts of education and schools in resistance, and, the Learn at Home Program, which presents the speeches of supporters and critics of its implementation. Updating in the report, the forms of pedagogical and ethical political action with which teachers have faced this unprecedented socio-educational condition, offers a framework to rethink the reconfiguration of the relationship between school agents and families, to fight for greater equity in the education system.

Key words: teachers; basic education; pandemic; school work

\section{Resumo}

O estudo analisa o trabalho escolar de professores de escolas urbanas e rurais de pré-escolar e ensino básico de Chiapas, perante a emergência sanitária por COVID19. A pesquisa é do tipo hermenêutica qualitativa, realizada a partir de entrevistas a 22 professores. A análise permitiu a construção de três blocos temáticos: Através da ecrã. Carências, angustia e desconforto; O trabalho escolar. Contextos públicos e privados de ensino e escolas em resistência; e o Programa Aprende em Casa. Atualizar na memória, as formas de ação 
Lo escolar a través de la pantalla. Trabajo docente en Chiapas, México. I

Germán Alejandro García Lara, Soledad Hernández Solís, Irma Hernández Solís, Oscar Cruz Pérez, Jesús Ocaña Zúñiga y Carlos Eduardo Pérez Jiménez.

pedagógica e ético-política com que os professores têm desafiado essa condição socioeducativa inédita, oferece um cenário para repensar a reconfiguração da relação entre agentes escolares e famílias, na luta por uma maior equidade do sistema educacional.

Palavras-chave: professorado; ensino básico; pandemia; trabalho escolar

\section{Introducción}

La sociedad en su conjunto fue sacudida por la emergencia sanitaria derivada del COVID19, nadie esperaba que la contingencia fuese más allá de semanas o escasos uno o dos meses, resultaba inaudito y ajeno a todo lo vivido. El cierre de las actividades no esenciales, a tres meses del término del ciclo escolar 2019-2020, era insospechado, se mostraba incredulidad, que paulatinamente fue incertidumbre y zozobra ante la creciente infodemia de enfermedad y mortandad, consecuencia de políticas neoliberales que han reducido los sistemas sanitarios y de salud pública. En educación básica, primeramente, se suspendieron las clases y se implementó la modalidad de educación a distancia, con lecciones virtuales a través de la televisión, así como del Programa Aprende en casa, que integra recursos tecnodidácticos de apoyo a padres y madres de familia, docentes y alumnos/as. En Chiapas y Oaxaca, se implementó el programa Aprendiendo desde mi comunidad, con emisiones de contenidos educativos en lenguas originarias.

La Secretaría de Educación Pública (SEP) (2015) señala que cuenta con 173.000 escuelas en este nivel, de las cuales 125.552 no tienen servicio telefónico, 76.383 no poseen computadoras o no funcionan y 123.511 carecen de acceso a internet. El INEE (2019) informa que solamente el 24\% de docentes de nivel preescolar contaban con materiales didácticos digitales en sus escuelas y aulas; y únicamente el $28.1 \%$ tenían computadoras e Internet.

Tales datos, desde la perspectiva de la segmentación socioescolar (Murillo, 2016), supone un acceso diferencial de los sujetos a la escuela a partir de sus condiciones socioeconómicas de pertenencia. Al respecto, Trejo (2020) plantea: "Estamos frente a una doble brecha digital educativa, donde la desigualdad es el mayor freno (...), dentro y fuera de los centros escolares (...), además, no se cuenta con las competencias en el uso y aprovechamiento de las tic" (p. 125). En este contexto de suma desigualdad y escasa reflexión sobre la modalidad y los recursos utilizados, las prácticas educativas del profesorado se avizoran complejas y poco alentadoras para la construcción de aprendizajes en el alumnado.

A la par de la falta de acceso y las competencias de uso de las TIC, lo mismo que las limitaciones para su capacitación, se refieren críticas a las elevadas expectativas que se tienen de su aplicación (Barrón-Tirado, 2012). Sobre este mismo aspecto, Díaz Barriga (2020) plantea:

La virtualidad supone el riesgo de pérdida del vínculo presencial y puede generar tensiones por la sobreexposición de docentes y estudiantes, o por las dificultades para mantener la relación y la mediación pedagógicas. Esto es especialmente cierto en los niveles iniciales, en los que se requiere un trabajo coordinado con padres, madres o cuidadores. (p. 25) 
Lo escolar a través de la pantalla. Trabajo docente en Chiapas, México. I

Germán Alejandro García Lara, Soledad Hernández Solís, Irma Hernández Solís, Oscar Cruz Pérez, Jesús Ocaña Zúñiga y Carlos Eduardo Pérez Jiménez.

Tal argumento es compartido por la CEPAL (2020) que expone: "Desde el punto de vista pedagógico, la virtualidad (...), requiere un trabajo coordinado con padres, madres o cuidadores para el acompañamiento y la mediación de los procesos de niños y niñas" (p. 11).

Además de ello, los cuestionamientos abarcan también las estrategias implementadas para su operación y la sobrevaloración del uso de material didáctico situado casi exclusivamente en los libros de texto: "se implementó una estrategia de aprendizaje rígida, acartonada, soporífera y con poca imaginación pedagógica; [como si...] no fuera necesario construir el propio conocimiento a través de tensar el pensamiento" (Ponce, 2020, p. 123).

Contreras (2020) señala que, para enriquecer y diversificar su enseñanza, profesores y profesoras proponen materiales didácticos accesibles al estudiantado y padres y madres de familia, elaboran su planeación didáctica y asisten regularmente a la escuela o comunidad, manteniendo comunicación cotidiana a través de la aplicación de mensajería instantánea telefónica de WhatsApp.

Las tareas realizadas por el equipo docente incluyen la planeación didáctica; el diseño de actividades para su ejecución autodidacta por el alumnado o con el apoyo de sus familias; la selección de recursos didácticos de bajo costo; la impartición de clases; el envío, recepción y calificación de tareas; así como todas las actividades organizativas para la evaluación de cada estudiante y la conformación de evidencias del trabajo propio y del alumnado. Aunado a ello, participa en reuniones colegiadas con compañeros/as y directivos/as y otras más de carácter organizativo con las familias. No obstante, existen profundas limitaciones en sus prácticas educativas, derivadas de las condiciones contextuales en que llevan a cabo su labor.

Hay quienes establecen comunicación a través de dispositivos y aplicaciones que tienen mayor conocimiento, como WhatsApp o Facebook; otras personas refieren dificultades para mantener la comunicación por estas u otras vías. Por otra parte, existe un desigual acceso a recursos y habilidades tecnológicas que posibilite ampliar y enriquecer la experiencia de aprendizaje a través del diseño de videos, videollamadas telefónicas, uso de plataformas digitales y otros recursos, aunque es patente el entusiasmo y disposición para el encuentro con familias y alumnos/as, así como a la revisión de los trabajos realizados (Meixueiro, 2020).

A continuación, se describen algunas de las experiencias reportadas en diversos países a partir de la aplicación de la modalidad a distancia o en línea. Baptista et al. (2020) reportan, en una encuesta realizada a 2.253 maestros de diferentes regiones de México, de los cuales 90 eran de nivel preescolar, que la comunicación con su alumnado se realiza a través de llamada a celular, teléfono fijo, videollamada o WhatsApp, mientras que, con las familias, es mediante correo electrónico, video llamada o WhatsApp. El uso de herramientas digitales más recurrentes son la radio y videos y, en cuanto a herramientas no digitales, se consideran predominantemente las fotocopias.

En un estudio llevado a cabo con familias y maestros/as de preescolar en Medellín, Colombia (Herrera et al., 2020), el equipo docente señala que existe acentuado ausentismo de los padres y madres y que la comunicación con ellos se realiza vía telefónica y a través de cuadernos. 
Lo escolar a través de la pantalla. Trabajo docente en Chiapas, México. I

Germán Alejandro García Lara, Soledad Hernández Solís, Irma Hernández Solís, Oscar Cruz Pérez, Jesús Ocaña Zúñiga y Carlos Eduardo Pérez Jiménez.

En Argentina, el personal docente de preescolar utiliza, sobre todo, tecnologías de redes sociales y recursos digitalizados (Expósito, 2020). Para ello, se ha promovido el acceso a contenidos pedagógicos, por diferentes medios de comunicación, y tecnológicos, se ha capacitado a docentes en el uso de TIC para la enseñanza remota y en los cambios para la evaluación y promoción de alumnos; además de ello, se han establecido convenios con empresas de telecomunicaciones para "permitir la navegación gratuita en las plataformas digitales educativas" (Cardini et al., 2020, p. 3). Ceballos y Sevilla (2020), en un trabajo realizado con docentes y familias de Córdoba, Argentina, plantean que la mayor parte de ellos utiliza WhatsApp y solo unas pocas plataformas virtuales: "Las docentes emplean estos medios para enviar fotos de sus carpetas, archivos Word y otras actividades en PDF" (p. 9). En Brasil, la investigación de Silva et al. (2020), con docentes de zonas rurales, destaca la precariedad de infraestructura, acceso a las TIC y alimentos en las comunidades, lo que conlleva a un limitado quehacer docente.

En países no latinoamericanos, trabajos como el de Fagell (2020), llevado a cabo en Estados Unidos, señalan una sobredemanda de los familiares hacia maestros/as por las tareas y el acompañamiento hacia el alumnado en diferentes horarios del día. En Noruega (Bubb y Jones, 2020), uno de los países que tiene un nivel de ingresos más altos per cápita en el mundo, refieren que la pandemia fue una oportunidad para la innovación, una enseñanza más creativa y una mayor independencia del estudiantado. En la Unión Europea, algunos de los principales hallazgos en investigaciones llevadas a cabo en el entorno de la pandemia, señalan que, con el inicio de la educación remota, se utiliza un menor tiempo para la enseñanza, destacan diferencias en la infraestructura digital entre las diferentes escuelas y de habilidades del personal docente para el manejo de tecnología en la modalidad a distancia (Di Pietro et al., 2020).

Otro aspecto que se aúna a la complejidad de la acción educativa ante la emergencia sanitaria es que profesores y profesoras - como el resto de la población- vivencian enfermedades o fallecimiento de familiares y amistades por contagio del COVID-19, crisis familiares, sociales y pobreza, lo mismo que mayores niveles de violencia doméstica, problemas de salud física y malestar psíquico, aunado a la falta de recursos materiales o profesionales para la realización de su trabajo (CEPAL, 2020).

Llama la atención que el contexto sociocultural y económico, de las condiciones materiales de vida y la cultura en que se desarrollan los sujetos, no se enfatice como un aspecto decisivo para la actividad escolar; aspecto de particular relevancia en Chiapas, uno de los Estados más pobres de México y con amplia diversidad cultural. En este sentido, el presente estudio tiene como propósito analizar el trabajo escolar de docentes de preescolar y primaria en Chiapas, ante la emergencia sanitaria por COVID-19.

\section{El proceso metodológico}

El estudio se realiza desde una tradición cualitativa, para la cual el análisis de la realidad es inseparable del contexto, se profundiza en los significados que la gente atribuye y construye de dicha realidad, en que las interpretaciones son construcciones intersubjetivas de los fenómenos 
de la vida cotidiana, social e históricamente condicionada (Rodigou y Paulín, 2013). En específico, en el ámbito educativo, penetra en la comprensión de los fenómenos, la valoración de la práctica educativa y la toma de decisiones (Sandín, 2003).

El método utilizado es el hermenéutico, el cual incluye "no solo el análisis gramatical, sino también un análisis del contexto histórico de cualquier acontecimiento" (Sandín, 2003, p. 59). Para ello, se considera la hermenéutica crítica, en la cual "lo importante es la valoración de los significados de dicha expresión a la luz de las condiciones históricas" (Sandín, 2003, p. 61).

\section{Técnicas e instrumentos}

Se aplicaron entrevistas semiestructuradas, cuyo propósito fue el de "obtener descripciones del mundo de la vida del entrevistado con respecto a la interpretación del significado de los fenómenos descritos; tiene una secuencia de temas que se han de cubrir, así como algunas preguntas propuestas" (Kvale, 2011, p. 79-80). De inicio, se obtuvieron datos generales de los entrevistados como edad, escolaridad, horario de trabajo, escuela en que labora, colonia en que se ubica la escuela, años de servicio y número de alumnos/as que atiende. Se consideró una guía de entrevista: "herramienta de trabajo reflexivo para la organización de los temas posibles que pueden aparecer en la conversación" (Galindo, 1998, p. 316), la cual se integra a continuación.

A partir del contexto en que realiza su trabajo, ¿qué puede contarme sobre la actividad escolar que ha realizado en este periodo de emergencia sanitaria por COVID-19?

Pudiera explicarme, ¿qué tipo de información, indicaciones o asesoría brinda a las familias o alumnos/as?

En términos didácticos, ¿cómo explicaría el desarrollo de su trabajo en esta modalidad?

Además, se analizaron archivos enviados por las docentes en los que se registran las actividades que deben realizar las familias y, específicamente, el alumnado para su aprendizaje.

\section{Participantes y contexto}

En Chiapas, el 76.4\% de su población vive en situación de pobreza (CONEVAL, 2020); el $51,27 \%$ reside en zonas rurales y un $27,99 \%$ habla una lengua indígena, sectores que, en su conjunto, presentan las mayores carencias sociales (INEGI, 2020). Solamente el $73.1 \%$ de personas de zonas urbanas y el $40.6 \%$ de zonas rurales tienen conectividad digital, menos del $60 \%$ del total de su población (IFT, 2020). La matrícula escolar en la entidad es de 1.788 .859 alumnos/as, atendidos/as en 19.414 escuelas; de estos, $94.10 \%$ asisten a escuelas públicas y $5.88 \%$ a escuela privadas. En preescolar, se cuenta con 295.194 alumnos/as, de los/as cuales $60,60 \%$ asiste a escuelas de tipo general, que considera distintas formas de organización y ubicación; 30,64\%, indígena y 8,73\%, comunitaria. En primaria, se cuenta con 788.919 alumnos/as, de los/as cuales $65,35 \%$ asisten a escuelas de tipo general; $31,31 \%$, indígena y 3,32\%, comunitaria, lo que aprecia una estructura multiforme y heterogénea en la que tres de cada diez alumnos/as demandan una 
educación adecuada a su lengua y características. Para su atención, laboran 85.833 docentes, $87,07 \%$ en el sector público y $12.96 \%$ en el sector privado (Mérida y Acuña, 2020) y, en el grueso de este, milita uno de los bloques más importantes de la disidencia magisterial de México, con una acción político-educativa tendiente a agenciar mejores condiciones laborales, la democratización del sindicato y una educación de mayor equidad.

Las y los docentes que participan en el estudio son 22: 18 mujeres y cuatro hombres, con edades de 24 a 54 años. Seis cursaron estudios de posgrado y el resto de licenciatura en diferentes programas de educación. Las características de los centros escolares en que laboran se exponen en la Tabla 1. Las escuelas de organización bidocente y multigrado bilingüe no cuentan con docentes de apoyo.

\section{Tabla 1}

Caracteristicas de los centros escolares y ubicación

\begin{tabular}{|c|c|c|c|c|c|c|c|}
\hline $\begin{array}{l}\text { Tipo de } \\
\text { escuela }\end{array}$ & Nivel & Sector & Municipio & $\begin{array}{l}\text { Región de } \\
\text { Chiapas }\end{array}$ & $\begin{array}{l}\text { Número } \\
\text { de } \\
\text { docentes }\end{array}$ & $\begin{array}{l}\text { Número } \\
\text { de } \\
\text { escuelas }\end{array}$ & Organización \\
\hline \multirow[t]{7}{*}{ Rural } & Preescolar & Público & Acala & IV. De los Llanos & Dos & Una & Completa \\
\hline & & Público & Villacorzo & VI. Frailesca & Una & Una & Completa \\
\hline & & Público & Trinitaria & XV. Meseta & Una & Una & Bidocente \\
\hline & & & & Comiteca & & & \\
\hline & & & & Tojolabal & & & \\
\hline & Primaria & Público & Chiapa de & Metropolitana & Dos & Dos & Completa \\
\hline & & & Corzo & & & & \\
\hline \multirow[t]{5}{*}{ Indígena } & Primaria & Público & Chilón & VI. Selva & Tres & Una & Multigrado \\
\hline & & & & & & & bilingüe tseltal \\
\hline & & Público & Las & XV. Meseta & Uno & Una & Multigrado \\
\hline & & & Margaritas & Comiteca & & & bilingüe \\
\hline & & & & Tojolabal & & & tojolabal \\
\hline \multirow[t]{7}{*}{ Urbano } & Preescolar & Público & Tuxtla & Metropolitana & Cinco & Cuatro & Completa \\
\hline & & & Gutiérrez & & & Una & Bidocente \\
\hline & & Privado & Tuxtla & Metropolitana & Tres & Una & Completa \\
\hline & & & Gutiérrez & & & & \\
\hline & Primaria & Privado & Tuxtla & Metropolitana & Cuatro & Una & Completa \\
\hline & & & Gutiérrez & & & & \\
\hline & & & & Total: & 22 & 14 & \\
\hline
\end{tabular}

\section{Procedimiento}

El estudio fue avalado científica y éticamente por la Dirección de Investigación y Posgrado de la Universidad de Ciencias y Artes de Chiapas. Para el trabajo, se contactó al personal docente, a quienes se informó sobre el estudio y se les invitó a participar. A quienes accedieron a colaborar, se les solicitó su consentimiento informado y se les indicó que la información se trataría de forma 
Lo escolar a través de la pantalla. Trabajo docente en Chiapas, México. I

Germán Alejandro García Lara, Soledad Hernández Solís, Irma Hernández Solís, Oscar Cruz Pérez, Jesús Ocaña Zúñiga y Carlos Eduardo Pérez Jiménez.

confidencial, exclusivamente con fines de investigación. Posteriormente, se acordaron las fechas de entrevista, las cuales se realizaron en su casi totalidad a través de plataformas digitales. En estas últimas, se asumen las limitantes de no contar con el acercamiento a las conductas no verbales del entrevistado y que el contexto sea el espacio virtual y no el de trabajo del sujeto (Denzin y Lincoln, 2015).

\section{Análisis de la información}

Se aplicó la técnica de análisis de información propuesta por Martínez (1998), para ello, se realizó la lectura reiterada de las entrevistas hasta contar con un panorama comprensivo de su contenido, se seleccionaron unidades de análisis o segmentos de discurso que se codificaron en categorías, se procedió a establecer su relación, comparación y reducción para intentar aprehender su sentido desde una perspectiva que toma en cuenta el contexto de su realización, integrándose en bloques temáticos más abstractos e incluyentes. Finalmente, se delimitaron los hallazgos y se realizó su interpretación contextual. Para la versión definitiva de los resultados del análisis, se llevó a cabo la retroalimentación comunicativa (Flick, 2007), con algunos/as de los/as docentes participantes, para considerar su acuerdo y punto de vista.

\section{Resultados}

El análisis de la información se estructuró en tres metacategorías: A través de la pantalla. Penurias, angustias y malestares, en que se aborda la percepción y las condiciones socioculturales en que los maestros y las maestras desarrollan su quehacer ante esta emergencia sanitaria; El trabajo escolar. Contextos públicos y privados de la enseñanza y escuelas en resistencia, que analiza diversos aspectos de su praxis, considerando de manera diferenciada a las escuelas del sector público, en las que, a su vez, se caracteriza a las escuelas denominadas en resistencia, en que se aplica el Programa de Educación Alternativa propuesto por la disidencia magisterial en Chiapas, y las del sector privado; y Programa Aprende en casa, instaurado por las autoridades educativas a partir de la emergencia sanitaria, en que se exponen los discursos de adeptos y críticos de su implementación, así como del uso de cuadernillos como opción para el trabajo escolar.

\section{A través de la pantalla. Penurias, angustias y malestares}

En las escuelas de Chiapas, México, las condiciones de operación son diversas. En las del sector público, pero sobre todo en aquellas de comunidades rurales, el equipo docente, las familias y el alumnado padecen la carencia de infraestructura y conectividad informática, a ello se agrega el que los padres y madres realicen otras tareas para sobrevivir y no cuenten con tiempo para apoyar a sus hijos e hijas, carezcan de recursos económicos, culturales y didácticos e, incluso, se encuentren desempleados. 
Lo escolar a través de la pantalla. Trabajo docente en Chiapas, México. I

Germán Alejandro García Lara, Soledad Hernández Solís, Irma Hernández Solís, Oscar Cruz Pérez, Jesús Ocaña Zúñiga y Carlos Eduardo Pérez Jiménez.

La actividad escolar devenida a partir de la pandemia significó, para todo el personal docente, una situación totalmente novedosa que implicó retos y cambios en el trabajo. La narrativa es la misma sin importar cargo, centro escolar o tipo de sector, aunque con matices distintivos.

Así, una de las entrevistadas de escuela pública y una de escuela privada, expresan su incertidumbre por el uso de las tecnologías de información y comunicación en la modalidad a distancia: "El mayor reto que se me presentó fue adaptarme a esta nueva modalidad. Al principio no sabíamos de qué manera íbamos a trabajar y ni cómo iban a responder los padres de familia" (Profesora Mirsa, preescolar rural, Acala); "Fue un gran reto, porque no estábamos acostumbrados a dar clases en línea, entonces sí fue un poco complicado, ¿cómo capacitarte?, ¿qué plataforma utilizar?, ¿cómo utilizarla?, ¿qué recursos utilizar?" (Profesora Iris, primaria, sector privado)

Tales narrativas "están vinculadas con un monto de angustia, ansiedad, malestar y sensación de 'extrañamiento' en relación con aquello que estábamos acostumbrados a hacer: ¿cómo hago yo ahora para dar o sostener las clases?" (Tranier et al., 2020, p. 8); pero también develan la precaria formación docente en competencias digitales y la carencia de dispositivos de este tipo (Navarrete et al., 2020).

Las condiciones socioculturales y económicas de las familias dificultan o facilitan un entorno proclive al aprendizaje, condiciones de segregación (Murillo y Martínez, 2017) que atestiguan la injusticia y desigualdad, superpuestas en los grupos y comunidades (Álvarez et al., 2020).

En las escuelas públicas rurales, ante la falta de recursos materiales y económicos de las familias, es común el uso de dispositivos de telefonía celular, a través de la aplicación de mensajería instantánea por WhatsApp; no obstante, debido a que esta requiere de internet, el envío o recepción de información no siempre puede llevarse a cabo. Otras aflicciones, como la enfermedad y la muerte de familiares, confluyen y reconfiguran las dinámicas familiares y escolares. "Nuestro recurso es el WhatsApp, porque otras aplicaciones para los padres de familia se les hace bastante difícil, por el manejo y el recurso, no todos cuentan con internet en casa, tienen que poner saldo y pagar" (Profesora Jania, preescolar rural, Villacorzo);

Los papás son de escasos recursos; se tiene que ir una vez por semana para poner tareas en libros, apoyándonos de varias actividades (...). Estamos trabajando por medio del WhatsApp, pero no he tenido la fortuna que todos los padres respondan. Con un niñito, desde octubre no he tenido comunicación, hace unos meses murió su papá y la señora es empleada doméstica. (Profesora Mariana, preescolar urbano)

Al respecto, pensar el uso de modelos a partir de tecnologías de la información en zonas en que se carece de posibilidades para su uso o los dispositivos para su implementación resulta una aspiración sin fundamentos. Además de ello, es indispensable la consideración sobre este tipo de recursos de enseñanza y su gestión en la vida y cultura escolar (Sunkel et al., 2014). "Es claro que no pudimos llevarlo de manera virtual, no hay acceso a internet y son pocas las familias que 
Lo escolar a través de la pantalla. Trabajo docente en Chiapas, México. I

Germán Alejandro García Lara, Soledad Hernández Solís, Irma Hernández Solís, Oscar Cruz Pérez, Jesús Ocaña Zúñiga y Carlos Eduardo

Pérez Jiménez.

cuentan con televisores, jes imposible trabajar de esa manera!" (Profesora Alicia, primaria indígena, Chilón);

Como en su casa [de uno de los alumnos] la luz se va todas las mañanas, su mamá se enteró un mes tarde del programa estatal de televisión que se transmite a esas horas. Su señal de televisión tampoco funciona bien cuando hay luz [...]; los docentes perciben mayor dificultad para realizar el trabajo en estas condiciones. (Profesora Noe, primaria rural, Chiapa de Corzo)

La formación escolar de las familias es, en algunos de los casos, muy limitada; además, por el trabajo que realizan, generalmente fuera de casa, restringen el apoyo a sus hijos e hijas, faltan a las reuniones o no responden a las comunicaciones del equipo docente, aspectos coincidentes con lo referido por Navarrete et al. (2020) y Sanz et al. (2020). "Las madres jno entienden!, en casa hay gente que no tiene estudios y isimplemente no comprende la actividad!, entonces hay que repetirlas una y otra vez en horarios extras, preguntándome si está bien o cómo lo van a desarrollar" (Profesora Magaly, preescolar urbano);

Los padres de familia no ayudan a sus hijos en la realización de sus actividades, porque muchos no saben leer ni escribir y no hablan el español y no podemos exigir de más porque realmente no pueden [...], la mayoría se dedican al trabajo del campo y es difícil poder apoyarlos. Hoy en día la comunidad nos exige regresar a las aulas. (Profesora Noemí, primaria indígena, Chilón)

Summer et al. (2020) destacan el aumento de la condición de pobreza en países como México y el limitado acceso a bienes que demanda la modalidad multimodal (Armitage y Nellums, 2020), inequidad que vulnera el derecho de acceso a la educación y a otros bienes como el de la salud en esta población.

En este contexto, el desánimo lacera las limitadas opciones que tiene cada docente para reinventarse en su quehacer. No basta acudir a las comunidades, idear nuevas formas de trabajo a través del diseño de actividades si padre y madres no se presentan o no responden y existe abandono escolar. En estos casos, el panorama es sombrío y se tienen limitadas expectativas respecto a los aprendizajes de cada alumno.

Cuando se suspendieron las clases en el ciclo anterior, asistían para entregar y recibir material, pero en este nuevo ciclo, de 22 alumnos, solo se inscribieron 13 y a partir de octubre comenzó la deserción escolar, al grado que el día de hoy solo tengo tres alumnos en el grupo de sexto grado, he visitado a los padres de familia, en algunas casas encontramos a los niños bajo llave porque los padres se van a trabajar, en otras las familias se fueron a trabajar sus tierras y se llevaron también a sus hijos para que trabajen. Con la deserción escolar, el desánimo en los estudiantes y el atraso académico que traemos arrastrando, el analfabetismo vuelve a tomar posesión por lo menos en las zonas rurales de Chiapas. (Profesor Javier, primaria rural, Chiapa de Corzo)

De 38 alumnos, solo quedan 24. Se está dando la deserción escolar tanto en mi grupo de primer grado como en los otros grados (Profesora Noe, primaria rural, Chiapa de Corzo). 
La penuria de estos alumnos y alumnas y familias, cuyo lugar de enunciación como ciudadanos/as resulta estéril, les instala como exiliados de la lógica de mercado y del capital; para ellos/as, toda acción pedagógica se estrecha y resulta una fabulación si no se acompaña de justicia social. Las escuelas de estas comunidades, al alcanzar menos logros que las demás, obtienen menos apoyos, lo que les mantiene segregadas e invisibilizadas (Tranier et al., 2020), lo que racionaliza la irracionalidad de las políticas que perviven en el sistema.

\section{El trabajo escolar. Contextos públicos y privados de la enseñanza y escuelas en resistencia}

\section{Atisbos a las escuelas de Chiapas}

El trabajo sigue una modalidad a distancia o en línea, conjuntamente al acompañamiento presencial, con el riesgo latente a su salud, semanal o quincenal para la revisión de tareas, el dejar otras más para un siguiente periodo, brindarles información o acordar diferentes acciones con ellos.

El proceso de atención para los padres de familia es de manera virtual y presencial, tengo un grupo de WhatsApp con los padres de familia en el cual envío las actividades correspondientes a cada semana, videos, fotografías, audios de las actividades, igual, cada padre de familia puede plantearme sus dudas sobre la realización de las actividades y también es presencial, ya que para hacer una evaluación requiero que me lleven físicamente las actividades que se les ha enviado. (Profesora Mirsa, preescolar rural, Acala);

Asistimos a la escuela una vez por semana dejando actividades tanto en los cuadernos y los cuadernillos, explico las actividades en tseltal y español que van a realizar durante la semana y así sucesivamente, aunque muchos de los alumnos no realizan todas las actividades. (Profesora Noemí, primaria indígena, Chilón).

La presencialidad periódica coloca al cuerpo del docente como "objeto de suplicio" (Tranier, 2021ª), lo que no es más que ceder, ante la presión social o política, al sometimiento y doblegamiento a través de diversos mecanismos de control de la autoridad, reificación de la relación laboral o bien como corporeidad autoexaltada de sus propias ausencias. Considerar formas alternas de presencialidad y evitar la intrusión de lo laboral al ámbito personal (de la salud, del tiempo de trabajo) es el eje sobre el cual tramitar la reflexión de las continuidades o rupturas en el ámbito administrativo pedagógico del docente.

Es común que no se mantenga con algunas familias la ya exigua comunicación a través de mensajería telefónica, aun así, se intenta conservar dicha comunicación simultáneamente con la compilación de evidencias diarias, semanales o mensuales; lo que, en el fondo, señala la necesidad comunicacional y afiliativa con padres, madres y alumnos/as, situación que es también referida por Álvarez et al. (2020). Dicho cúmulo de evidencias, en ocasiones, es más una demanda de la hipervigilancia, del panóptico en que la escuela se ha convertido, de modo que la presencialidad 
Lo escolar a través de la pantalla. Trabajo docente en Chiapas, México. I

Germán Alejandro García Lara, Soledad Hernández Solís, Irma Hernández Solís, Oscar Cruz Pérez, Jesús Ocaña Zúñiga y Carlos Eduardo

está sujeta perversamente a las evidencias, como si esta corroborara la existencia y certidumbre del quehacer docente.

La supervisora nos está monitoreando: "¿qué actividades van a subir?", nos pide evidencias, fotografías. Si llegamos una vez por semana y nos entrevistamos con los padres de familia, nos pide que tomemos cuando menos una foto, también tenemos que mandar una foto donde estamos en la computadora haciendo la videollamada con los niños. (Profesora Magaly, preescolar urbano).

Ante las condiciones socioculturales y económicas de las comunidades y de los/as propios/as docentes, la innovación, la creación de propuestas de trabajo escolar ha sido la cimiente para repensar la enseñanza de los contenidos y hacer significativo el aprendizaje a los alumnos.

Tienes que ir buscando actividades que sean creativas, Ilamativas, algo que puedan hacer de algún material que puedan tener en casa, que no tengan que gastar, que sean materiales reciclados, donde ellos puedan utilizar su imaginación, su creatividad. No forzosamente el hablar de tecnología se basa en las computadoras, el teléfono, las tecnologías pueden ser algo que el niño pueda construir, crear con los materiales. (Profesora Mariana, preescolar urbano);

Diariamente me comunico a través de un grupo de WhatsApp, les mando videos, cuentos, explicaciones sencillas, breves de cómo se va a realizar la actividad (...), trato de explicar mucho, de hacerles actividades interactivas de juegos de mesa, rompecabezas, loterías y con dados; en educación física, procuro respetar siempre el día en que se realiza la clase, tratando de que sea divertida. (Profesora Laura, preescolar urbano).

Las compañeras de tercero le están echando los kilos, hay un grupo que dos veces por semana se conectan por Zoom y empezó con cuatro chiquillos, ahorita ya la mayoría del salón, es otra experiencia para ellos de cómo aprender (Profesora Glendy, directora de preescolar urbano).

El equipo docente ha estado más que presente en su actividad pedagógica, ha restringido su presencialidad, pero no ha estado ausente (Tranier, 2021ª). Como profesional subalterno y antagónico, a través de su práctica, ha atenuado la crisis educativa y contrarrestado la tensión social ante el distanciamiento entre el Estado y las familias, cuya agencia en la arena política configura nuevos paisajes de lo educativo.

En este sentido, es posible afirmar que el equipo docente representa una potencia política y cultural en la intervención, cuya función ha sido crear discontinuidades y reconfiguraciones de su práctica (Tranier, 2021 ).

Esta actividad no ha sido tersa, se acompaña de dificultades de diversa índole, desde el tiempo que disponen los padres y madres o tutores/as, la organización, el seguimiento, los conocimientos, disposición y cualidades para compartir las consignas, así como las respuestas del alumnado, todo ello es un amasijo que condensa el acto educativo de acompañamiento. Los claroscuros de este proceso alientan ciertas notas de revancha sobre el reconocimiento social de la profesión, motivo de pugnas y desencuentros entre docentes y familias. 
Lo escolar a través de la pantalla. Trabajo docente en Chiapas, México. I

Germán Alejandro García Lara, Soledad Hernández Solís, Irma Hernández Solís, Oscar Cruz Pérez, Jesús Ocaña Zúñiga y Carlos Eduardo Pérez Jiménez.

Ahorita, de los papás es la responsabilidad para con sus hijos, para que aprendan; hacen el comentario que se les está complicando mucho puesto que no estaban acostumbrados ellos a dar clases (...), ahorita están comprendiendo que el trabajo que desempeña el maestro iqué loable es!, porque atiende a 30 a 35 niños y ellos con dos o tres en casa no pueden, se les hace complicado, ahorita vienen a valorar el trabajo del maestro o la maestra. (Profesora Magaly, preescolar urbano).

Evidentemente, no se trata de la falta de costumbre por la tarea de enseñanza escolar, es más bien la impostación de una función por la cual se le paga a otros, pero también de la falta de reconocimiento social de la profesión y de las profesoras y los profesores.

Para el profesorado, resulta innegable el efecto que la modalidad educativa tiene en su alumnado. Para algunos/as, es recurrente la mención a la falta de aprendizajes significativos relacionados con los contenidos del programa del nivel educativo correspondiente, lo cual es más prominente en contextos rurales e indígenas, pero también en zonas urbanas con condiciones de marginalidad y carencia de recursos, territorios expulsados del apoyo del Estado y sus instituciones.

Trabajamos un poquito más con el lenguaje oral, porque es donde vemos más las dificultades de los niños, como son muy pequeños su lenguaje oral es muy pobre, entonces tratamos de enviar actividades con rimas, con cantos, con poesías, con rondas, con juegos con exposiciones, con la finalidad de que el niño hable y hable. (Profesora Jania, preescolar rural, Villacorzo).

Destacan, además, aspectos socioafectivos, de desarrollo de procesos lingüísticos, entre otros:

Les está afectando, pues realizan las actividades solos en casa. La convivencia, el relacionarse, la interacción con sus demás compañeros facilita que el aprendizaje sea mucho más amplio, porque entre ellos se comparten ideas, observan cómo lo hacen sus compañeros y van aprendiendo. En casa [con alumnos de] primer grado, se da la sobreprotección de los papás, siguen pensando que son pequeños y que no lo pueden hacer; cosas que descartamos cuando entran al jardín, la docente se enfoca en que el niño sea totalmente autónomo, que aprenda a hacer las cosas por sí solo. (Profesora Jania, preescolar rural, Villacorzo).

La relación entre docentes y estudiantes es casi nula, aun así, les es posible identificar cualidades en ellos, al igual que algunas limitantes en su desarrollo socioafectivo. La falta de contacto físico con el alumnado, tan privilegiado en la cotidianidad escolar, es el aspecto nodal que circunscribe el acto pedagógico de encuentro, de andamiaje de los procesos de enseñanza y aprendizaje y, con ello, de subjetividades (Tranier et al., 2020).

No conozco a mi niño [se ríe] ino nos conocemos!, siento sentimientos muuuy así de frialdad; por ejemplo, ya se les ha dicho a los papás que no lleven a sus hijitos [a las reuniones], pero ahí va asomando alguna mamá con las criaturas, que cuando ven a su maestra, viene la niña con su cubre bocas y se lo quita: "maeeestra", le dice y mi amiga le 
Lo escolar a través de la pantalla. Trabajo docente en Chiapas, México. I

Germán Alejandro García Lara, Soledad Hernández Solís, Irma Hernández Solís, Oscar Cruz Pérez, Jesús Ocaña Zúñiga y Carlos Eduardo Pérez Jiménez.

hace así con las manitas [como deteniéndola]: "jespérate, mamita, espérate, mamita!, así, de lejos", iqué cruel, qué cruel! (Profesora Glendy, directora de preescolar, zona urbana). Siempre lo he dicho, jno va a ser igual!, tenerlos ahí, a través de la pantalla, en preescolar, lo que llegan a afianzar son amistades y lo que le motiva es ver a sus amigos. Se da una conexión bien bonita entre docente y niño, jmuy, muy bonita! y eso ¡se extraña, la verdad! (Profesora Mayra, preescolar urbano, sector privado).

En este entorno tan complejo, también obtienen logros en diferentes ámbitos, como en la relación que han logrado establecer con las familias y alumnos y alumnas, en el diseño de actividades innovadoras, el uso de tecnologías, pero, sobre todo, de aprendizajes que parecen distantes de alcanzar.

El grueso de profesores en el nivel preescolar son mujeres, con toda la implicación que culturalmente se ha depositado en ellas respecto del cuidado y apoyo en la crianza infantil. Esta condición también se expresa en la complejidad de su quehacer como docente y como madre de familia.

En preescolar, la mayoría somos mujeres, como compañeras, debemos ponernos en el lugar de los papás [...], mucha gente está teniendo síntomas aislados, algunas no llegan, pero la vecina le lleva el cuadernillo a la que no pudo ir porque tiene diarrea, otras que tienen fiebre, otras que tienen unas gripas tremendas, entonces aparece solamente un síntoma de varios que son asociados a esta enfermedad, ahí está el lazo de la hermandad, les digo: "isomos mujeres!". (Profesora Glendy, directora de preescolar, zona urbana).

En este tipo de situaciones, es posible "reinaugurar (y no cancelar) la solidaridad social a través del amparo y del cuidado: dos caras capaces de contrarrestar verdaderamente a la muerte (no en su sentido biológico, sino fundamentalmente político y subjetivo)" (Tranier et al., 2020, p. 10).

\section{Las escuelas en resistencia}

En Chiapas, la Coordinadora Nacional de Trabajadores de la Educación [CNTE], surgida desde 1979, forma parte de la disidencia magisterial de México. Para la educación preescolar, ha propuesto el Proyecto de Educación Alternativa (PEA), el cual utilizan algunas escuelas y docentes como referente para el trabajo cotidiano. En este, incluyen de manera integrada las líneas formativas y el plan escolar y comunitario (PEC). Así, la autodefinen: "Nuestra escuela es una escuela en resistencia y pertenecemos al bloque democrático de la sección siete, los programas que marca la SEP, de la nueva escuela mexicana, pues no lo estamos abordando, estamos abordando el Proyecto de Educación Alternativa" (Profesora Jania, preescolar rural, Villacorzo). Sobre su estructura y aplicación, narra una docente:

Elaboramos un proyecto de educación alternativa una vez al mes durante todo el ciclo escolar y finalizamos, en el mes de julio, con una feria de aprendizaje, es un plan escolar comunitario basado en talleres, una semana trabajamos el taller sobre un tema y 
proyecto, lo elaboramos a nivel escuela, para todos los grupos las actividades son las mismas, lo único que cambia es el grado de dificultad; dos semanas trabajamos a partir de la planeación con un libro que propone la Sección 7 que es el PEA, de acuerdo a la necesidad que nosotros vemos en el grupo [...], para todas es igual el método de enseñanza [...], primero mandamos la descripción de la actividad y lo que queremos favorecer, les enviamos un pequeño vídeo donde explicamos a los niños cómo lo vamos a jugar, qué tenemos que hacer; igual para los papás, también mandamos videos de cantos y de juegos, porque en el nivel de preescolar la estrategia es lúdica; y la última semana, para concluir el mes, trabajamos con un cuadernillo que son engargolados de fotocopias que nosotras elaboramos, nos ayudan para favorecer lo que es la motricidad fina, con el coloreado, delineado, el recorte de figuras, manejo de la tijera y todo ese tipo de cosas que son de manera manual. (Profesora Jania, preescolar rural, Villacorzo).

Díaz Barriga (2020) expone que el método de proyectos es útil para gestionar los aprendizajes de los alumnos y de una escuela que reflexiona sobre sí y sus alcances en el que: "en vez de pensar los contenidos desde su organización en las disciplinas, es poner éstas al servicio de lo que la realidad está reclamando" (p. 27).

Los y las docentes que militan en estas escuelas asumen una postura crítica sobre la modalidad en línea y el uso de las TIC. Respecto a la función de las familias en la tarea que se les ha responsabilizado ante la emergencia sanitaria, reconocen el enorme esfuerzo que realizan en este proceso, aunque también exaltan la acción profesional de cada docente.

Los objetivos no están siendo cumplidos porque la tecnología nunca va a sobrepasar la pedagogía del maestro. La intervención que tiene papá o mamá en casa no es la misma que podría tener el maestro con los alumnos, la paciencia, el análisis, la observación; realmente maestro es quien estudia la carrera y quién puede de verdad detectar cuáles son los errores o las estrategias que debe utilizar en el aula. (Profesora Jania, preescolar rural, Villacorzo).

No obstante, mantienen las mismas problemáticas y condiciones que las referidas para otras escuelas del sector público respecto a la participación de las familias, la recepción de evidencias del trabajo realizado, del proceso de evaluación, aunque su preocupación no deviene de la supervisión o vigilancia que sobre ella establecen las autoridades escolares, sino por el aprendizaje que los alumnos han o no alcanzado.

\section{Las escuelas del sector privado}

En el estudio, se entrevistaron a docentes de dos escuelas del sector privado, una del nivel preescolar y otra del nivel primaria, ambas de Tuxtla Gutiérrez. En estas, se trabaja con la modalidad en línea, ya que tienen a su disposición lo mismo que las familias, el acceso a internet y diversos medios y plataformas tecnológicas, de las cuales utilizan Zoom, Meet, ClassDojo y Kahoot. 
La planeación didáctica se elabora de una a dos semanas. Todos los materiales son proporcionados por las familias. Las sesiones diarias se intentan desarrollar lo más parecido posible al esquema que cotidianamente realizaban, aunque los tiempos se han reducido por lo que su clase es de una a dos horas y el resto de la jornada la enseñanza la realizan docentes de educación física, educación artística, inglés y computación.

Aunque para ellos/as el trabajo en esta modalidad es totalmente nuevo, han podido establecer formas de organización grupal y actividades para eficientar el trabajo y que este sea más dinámico y atractivo para alumnos y alumnas.

Organizo el grupo, que no solo estén sentados y estén en constante movimiento, entonces los niños como que están más activos, ya al final si ellos quieren expresar algo, darles la oportunidad. Aparte, se hacen dos grupos, dependiendo de las características de los alumnos y eso se ha funcionado (...), también el utilizar los juegos digitales, escucharlos y hacer que participen de algunos temas de su interés. (Profesora Martha, preescolar); Lo que yo hice fue preparar presentaciones en PowerPoint, Prezzi, que fuera un poquito más dinámico y precisa la explicación, por medio de Zoom se compartía la pantalla y presentaba la clase a los chicos y las páginas que teníamos que trabajar, a mí me funcionaron mucho las presentaciones la búsqueda de videos en Youtube. (Profesor Luis, primaria).

En este proceso organizativo, el apoyo de las familias ha sido de suma importancia. Realmente admiro mucho a esos papás que dejan que sus hijos trabajen solos; o sea, sí están ahí con ellos, sí se sientan al lado y trabajan con ellos, pero en ningún momento se meten a la clase o intervienen cuando el niño está participando o les estoy explicando. (Profesora Ada, primaria).

El aprendizaje de una nueva gramática que se recupera de las narrativas, acciones y silencios de padres y madres insinúa otras escenificaciones que disputan la verdad única atribuida al maestro/a (Tranier et al., 2020), pero que, paradójicamente, recuerda con más asombro y deseo a la escuela, sus espacios y su tiempo.

Para el proceso de evaluación de los trabajos, operan a través de correos electrónico o recursos con los que cuenta la propia plataforma. Los inconvenientes se relacionan con dificultades en la señal de internet, la intervención de algunos padres o madres de familia, mantener la atención del alumnado o la renuencia de estos a participar:

"Supe de otros compañeros que las mamás intervenían y hasta hacían comentarios ahora sí que jfeos con respecto a la clase o algo que la maestra comentaba!" (Profesora Ada, primaria);

El berrinche de que si no quieren hacer las cosas avientan todo y se van, y el papá así de que: "es que ya no quiso" y desconectan la cámara, prácticamente me dicen: "no lo voy a obligar", jse aburrió!; o me mandan un mensaje "no quiere entrar porque no se levanta". (Profesora Mayra, preescolar). 
Lo escolar a través de la pantalla. Trabajo docente en Chiapas, México. I

Germán Alejandro García Lara, Soledad Hernández Solís, Irma Hernández Solís, Oscar Cruz Pérez, Jesús Ocaña Zúñiga y Carlos Eduardo Pérez jiménez.

Los logros también son destacados por el profesorado de estas instituciones, en términos de los aprendizajes alcanzados y el uso de tecnología que enriquece el trabajo de enseñanza.

\section{Programa Aprende en casa}

La aplicación del programa Aprende en casa, implementado por la Secretaría de Educación, depende también del acceso a la televisión o internet y la disponibilidad de tiempo en los horarios asignados para la transmisión de contenidos a los diferentes grados escolares.

Al inicio del ciclo escolar, se nos indicó que vamos a trabajar con la estrategia de Aprende en casa dos. Consiste en que los niños observen la clase por televisión, dividido en dos clases de media hora diaria, donde se abordan los tres campos formativos y las tres áreas de desarrollo personal y social. La dinámica es que los padres de familia observen la televisión y realicen la retroalimentación que se les envía para cada clase. Por alguna situación que los padres de familia no cuenten con el tiempo, o que no se adapten al horario de la clase, también les mando el enlace de YouTube, algunos videos, cuentos, actividades, canciones interactivas, para fortalecer ese aprendizaje esperado enmarcado en la programación (...), la clase de inglés es la única que me ha parecido muy tediosa porque es para primer ciclo; es decir, para primero y segundo de primaria y también es la clase que se transmite para preescolar (...). No todos los padres de familia cuentan con wifi, solo algunos, en ese caso [solo ellos] pueden aprovechar la tecnología en casita para lograr aprendizajes en sus niños y que sea más llamativo, más interesante para ellos. (Profesora Laura, preescolar urbano).

Su prescripción formal y legal toma como principio condiciones de igualdad para la población, lo que a su vez constituye un entorno poco favorable a la inclusión (Sen y Kliksberg, 2007). En el ámbito curricular, se invisibiliza la diferencia de los sujetos de la educación y oculta la tensión entre lo común y lo diverso, no solo del alumnado, sino de las comunidades a las que pertenecen.

El programa Aprende en casa es una estrategia que a mí no me favorece y no siento que sea de utilidad; creo que es más para entretener, porque estando aquí en la misma ciudad ¡hay colonias donde no hay internet!, jhay padres de familia que no tienen televisión!, iy estamos en la ciudad! Por eso, nosotros, en nuestro jardín, buscamos otra estrategia de cómo enseñarles a los niños, porque primeramente algunos papás no cuentan con televisión y otra que en el tiempo de la mañana no está, la mayoría trabaja. En lo que respecta a preescolar (Aprende en Casa), pues se maneja un solo horario, el tema es para los tres grados y son diferentes en el día. (Profesora Mariana, preescolar urbano).

Para Mérida y Acuña (2020), la implementación del programa se funda en un acto político, mecanismo paliativo emergente para alcanzar los objetivos de aprendizaje propuestos en los planes y programas de estudio, no considera la diversidad económica y sociocultural del país, lo que acentúa la desigualdad social; no se arguyen, además, las certezas pedagógicas de su implementación. 
La alternativa ante tales carencias comunitarias y familiares y condiciones laborales y escolares de las propias familias fue el desarrollo de cuadernillos de trabajo.

Los cuadernillos surgen por la necesidad de poder trabajar con los alumnos a distancia porque realmente no hay acceso a internet en esta localidad y mucho menos televisores de prepago para que puedan llevar a cabo las clases en línea (...). La Secretaría de Educación manda cuadernillos para cada grado escolar, los contenidos son muy elevados a nivel de comprensión de los estudiantes, porque muchos no hablan el español, jes zona indígena!, son pocos los padres de familia que saben leer y escribir y se llegó a la conclusión, junto con directores y el supervisor de la zona, que no era prudente trabajar con esos cuadernillos, nos dimos a la tarea de realizar nuestras propias actividades de los mismos contenidos de todas las materias, lo adecuamos a nuestra planeación, la diferencia era hacer actividades menos complejas, con palabras más sencillas y que no necesitan de mucho apoyo. Citamos a los alumnos en la escuela, le explicamos en qué consisten las actividades tanto en español como tseltal. Entramos una vez por semana a recoger los cuadernillos y llevamos otro para que lo realicen durante la semana y así sucesivamente. (Profesora Noemí, primaria indígena, Chilón).

Trabajamos con cuadernillos que cada maestro elabora con el material que tiene, porque los cuadernillos que manda la SEP están muy complicados para los alumnos (Profesor Javier, primaria rural, Chiapa de Corzo).

Al respecto, Mérida y Acuña (2020) señalan que la región Altos Tsotsil Tseltal de Chiapaas son regiones con los más elevados niveles de pobreza y rezago, menor acceso a bienes que el programa demanda para el trabajo escolar, con lo que desigualdad y brecha educativa se mantiene e incluso se profundiza. En torno al uso casi exclusivo de cuadernillos, la cuestión es si con ello se logra integrar un currículum mínimo obligatorio. Otras experiencias exponen esto como un recurso paliativo ante la emergencia sanitaria (Álvarez et al., 2020).

Su uso, en algunas escuelas, se ha acompañado con las directrices del programa Aprende en casa, proceso en que la participación de las familias es crucial, como lo expresa una de las docentes:

Les hemos mandado cuadernillos, de acuerdo a los temas que pasan en Aprende en casa. No ha dado los resultados que quisiéramos, de mi grupo, un $70 \%$ que sí se comprometieron y el resto pues no me mandaron actividades, me comentaron que los niños ya se habían aburrido, que no querían ver la programación de la tele. (Profesora Mirsa, preescolar rural, Acala).

\section{Conclusiones}

El personal docente labora en centros de trabajo y comunitario con condiciones y recursos diversos. En aquellos que atienden a alumnas/os en escuelas del sector público, independientemente del tipo de organización del centro escolar: completa, bidocente o multigrado, las condiciones son sumamente precarias. En estos, excepto en uno de los casos, no 
Lo escolar a través de la pantalla. Trabajo docente en Chiapas, México. I

Germán Alejandro García Lara, Soledad Hernández Solís, Irma Hernández Solís, Oscar Cruz Pérez, Jesús Ocaña Zúñiga y Carlos Eduardo Pérez Jiménez.

utilizan plataformas digitales para el desarrollo de trabajos, por falta de conectividad digital en la comunidad o bien de recursos para el pago de servicios de internet o telefonía celular. Ante ello, han optado por el trabajo a través de mensajes vía WhatsApp o el uso de cuadernillos; algunos docentes se presentan semanal o quincenalmente en la comunidad para recibir o devolver trabajos elaborados por el alumnado o informar y acordar con las familias sobre diferentes aspectos del acontecer escolar. Preparan información digital o impresa, textos varios, videos, presentaciones en PowerPoint, lista de direcciones en Internet a las que pueden acceder para consulta de material, entre otras. El seguimiento se realiza casi exclusivamente a través de WhatsApp. Cada docente cubre los gastos de los materiales didácticos que utiliza, lo mismo ocurre respecto al uso de telefonía u otro dispositivo que utilicen.

Por otra parte, docentes del sector privado han mantenido, durante todo el periodo de emergencia sanitaria, el trabajo en línea a través de plataformas digitales, con un horario casi similar al ordinario, con periodos de tiempo para el alumnado en que se retiran de la conexión para realizar las tareas que les son asignadas. En la escuela de nivel preescolar, asisten a la institución y, en esta, les proporcionan equipo de cómputo y conectividad informática para realizar las actividades a través de la plataforma, mientras que, en la escuela primaria, esta actividad la realizan desde su hogar. En ambas escuelas, las y los docentes pueden acceder a material didáctico amplio, suficiente y diverso para el trabajo que realizan y se apoyan en otras/os profesionales en la jornada laboral. Las madres, padres o tutores de cada alumno acompañan diariamente en el trabajo que realizan.

El profesorado ha mostrado estar presente, incluso más allá de sus horas y límites en la revitalización creativa del currículum, de su adecuación a contextos y condiciones específicas, en el acompañamiento y diversificación de recursos y materiales didácticos, en el acompañamiento mutuo con las familias, en la casi insostenible necesidad de compartir los encuentros en formas más cercanas y efusivas, todo ello, es parte de su (re)configuración como profesional en una realidad inédita como la de la pandemia. Se ha constituido, ante las difíciles condiciones de trabajo, en referente para la tutoría, la orientación, la guía y el sostén de un vínculo necesario para procurar los aprendizajes (Muñoz y Lluch, 2020).

La oportunidad que ofrece esta realidad, de dejar de tributar en la provisión de conocimientos tan vituperada como el efecto más devastador de la vida escolar, ha ampliado la perspectiva de lo educativo, en esferas como la de los valores, de la participación escolar de las familias y su reincorporación como agente coadyuvante al desarrollo del alumnado en la cartografía pedagógica de las escuelas. La experiencia compartida muestra la dolorosa realidad socioeducativa en nuestra entidad, pero también irrumpe con fuerza en la discontinuidad al burocrático quehacer cotidiano de la docencia, le reencuentra allí, donde se han tenido que innovar acciones y ofrecer encuentros que transitan a través de una realidad virtual y en el desencanto y frustración por la ausencia del alumnado.

Son las alianzas políticas con las comunidades en que laboran los maestros (Tranier, 2021b), lazos que se mantendrán más allá de la pandemia; sobre todo en aquellos espacios, donde 
las y los maestros estuvieron, aun a riesgo de su salud, llamaron o enviaron mensajes, buscaron a las familias, les visitaron en sus viviendas y mantuvieron incólume la confianza en la escuela y sus agentes. En este intersticio, también se han tejido vínculos solidarios, de respeto, de colaboración, de reflexión sobre las formas de relación establecidas entre los agentes de las escuelas y las familias. Actualizar en la memoria estas formas de acción ético-políticas es lo que nos hace humanos y ofrece un sentido al trabajo docente (Tranier et al., 2020).

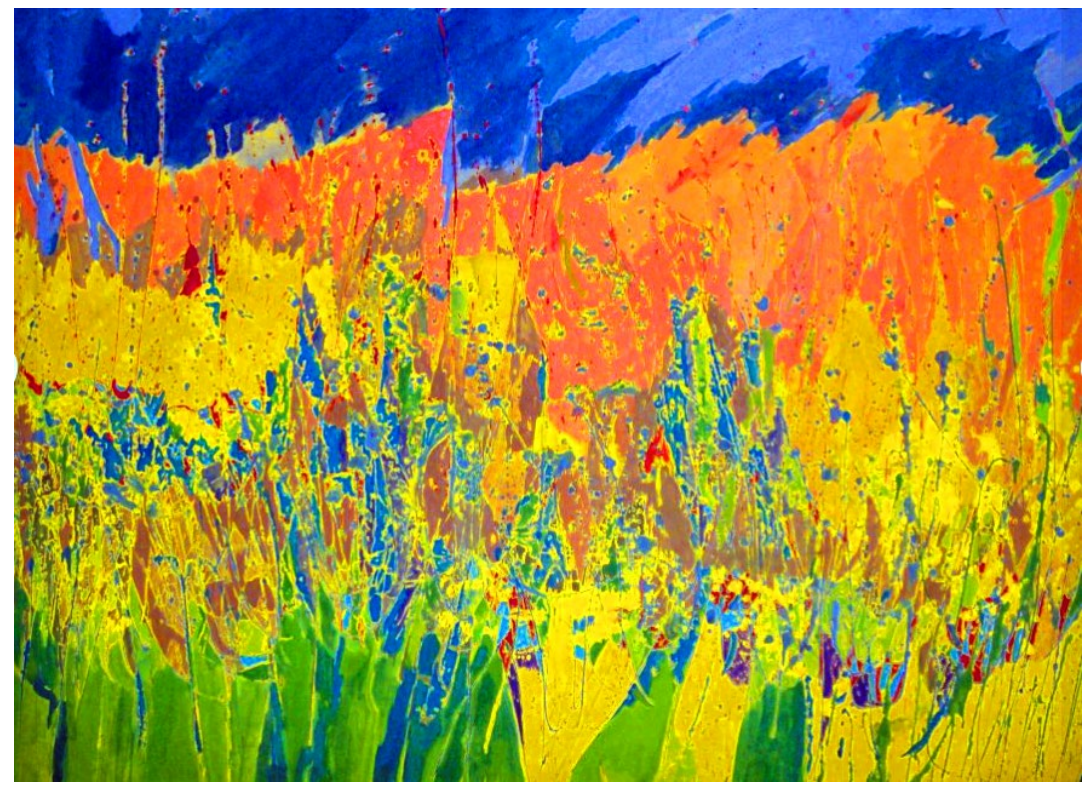

Paisaje sobre el Chimehuin, acrílico sobre papel. Paula Boh

\section{Bibliografía}

Álvarez, M., Gardyn, N., Iardelevsky, A. y Rebello, G. (2020). Segregación educativa en tiempos de pandemia: balance de las acciones iniciales durante el aislamiento social por el Covid-19 en Argentina. Revista Internacional de Educación para la Justicia Social, 9(3e), 25-43. https://doi.org/10.15366/riejs2020.9.3.002 Armitage, R. y Nellums, L. B. (2020). Correspondence. Considering inequalities in the schoolclosure response to Covid-19. The Lancet Global Health, 8(5), 644. https://doi.org/10.1016/S2214-109X(20)30116-9 Barrón Tirado, M. C. (2020). La educación en línea. Transiciones y disrupciones. En H. Casanova Cardiel, Educación y pandemia. Una visión académica (pp. 66-82). Instituto de Investigaciones sobre la Universidad y la Educación/UNAM.

Bubb, S. y Jones, M. A. (2020). Learning from the COVID-19 home-schooling experience: Listening to pupils, parents/carers and teachers. Improving Schools, 23(3), 209-222. https://doi.org/10.1177/1365480220958797 Cardini, A., Bergamaschi, A., D’Alessandre, V., Torre, E. y Ollivier, A. (2020). Educar en pandemia: entre el aislamiento y la distancia social. Banco Interamericano de Desarrollo. 
Lo escolar a través de la pantalla. Trabajo docente en Chiapas, México. I

Germán Alejandro García Lara, Soledad Hernández Solís, Irma Hernández Solís, Oscar Cruz Pérez, Jesús Ocaña Zúñiga y Carlos Eduardo Pérez jiménez.

https://publications.iadb.org/publications/spanish/document/Educar-en-pandemia-Entre-el-aislamientoy-la-distancia-social.pdf

Ceballos Marón, N. A. y Sevilla Vallejo, S. (2020). El efecto del aislamiento social por el Covid-19 en la conciencia emocional y en la comprensión lectora. Estudio sobre la incidencia en alumnos con trastornos de aprendizaje y menor acceso a las nuevas tecnologías. Revista Internacional de Educación para la Justicia Social, 9(3e), 1-13.

Baptista Lucio, P., Almazán Zimerman, A., Loeza Altamirano, C. A., López Alcaraz, V. A. y Cárdenas Domínguez, J. L. (2020). Encuesta nacional a docentes ante el COVID-19. Retos para la educación a distancia. Revista Latinoamericana de Estudios Educativos, L, 41-88.

CEPAL, OREALC Y UNESCO. (2020). Informe COVID-19 CEPAL UNESCO. La educación en tiempos de la pandemia de COVID-19. CEPAL/UNESCO.

Consejo Nacional de Evaluación de la Política de Desarrollo Social. (2020). Informe de pobreza y evaluación 2020. Chiapas. CONEVAL.

https://www.coneval.org.mx/coordinacion/entidades/Documents/Informes_de_pobreza_y_evaluacion_20 20_Documentos/Informe_Chiapas_2020.pdf

Contreras Arreola, L. (2020). En busca de la nueva normalidad educativa en escuelas rurales ante el COVID19. En J. Navarro Saras (Coord.), Los tiempos educativos en e/ Covid-19. Historias, vivencias y testimonios desde la escuela distante (pp. 189-194). https://revistaeducarnos.com/wpcontent/uploads/2020/07/covid19-3.pdf

Denzin, N. K. y Lincoln, Y. S. (2015). Manual de investigación cualitativa. Volumen IV. Métodos de recolección y análisis de datos. Gedisa.

Di Pietro, G., Biagi, F., Costa, P., Karpiński, Z. y Mazza, J. (2020). The likely impact of COVID-19 on education: Reflections based on the existing literature and recent international datasets. European Union.

Díaz Barriga, A. (2020). La escuela ausente, la necesidad de replantear su significado. En, H. Casanova Cardiel, Educación y pandemia. Una visión académica (pp. 19-29). Instituto de Investigaciones sobre la Universidad y la Educación/UNAM.

Expósito, E. y Marsollier, R. (2020). Virtualidad y educación en tiempos de COVID-19. Un estudio empírico en Argentina. Educación y Humanismo, 22(39), 1-22. https://doi.org/10.17081/eduhum.22.39.4214

Fagell, P. (2020). Coronavirus Shutdown. Phi Delta Kappa, 101(8), 7-68.

https://doi.org/10.1177/0031721720923799

Flick, U. (2007). Introducción a la investigación cualitativa. Morata.

Galindo Cáceres, J. (1998). Técnicas de investigación en sociedad cultura y comunicación. Pearson Addison

Wesley.

Herrera-Rivera, O., Álvarez-Gallego, M., Coronado-Mendoza, A. y Guzmán-Atehortúa, N. (2020).

Acompañamiento en educación inicial: voces de sus protagonistas en apertura al cambio. Educación y

Humanismo, 22(39), 1-31. https://doi.org/10.17081/eduhum.22.39.3858

Instituto Federal de Telecomunicaciones. (2020). En México hay 80.6 millones de usuarios de internet y 86.5

millones de usuarios de teléfonos celulares: ENDUTIH 2019. http://www.ift.org.mx/comunicacion-y-

medios/comunicados-ift/es/en-mexico-hay-806-millones-de-usuarios-de-internet-y-865-millones-de-

usuarios-de-telefonos-celulares

INEE. (2019). La educación obligatoria en México. INEE.

INEGI. (2020). Información por entidad. Chiapas. Población. INEGI. Recuperado el 4/7/2020 de

http://cuentame.inegi.org.mx/monografias/informacion/chis/poblacion/distribucion.aspx?tema=me\&e=07 
Kvale, S. (2011). Las entrevistas en investigación cualitativa. Morata.

Martínez, M. (2008). La investigación cualitativa etnográfica en educación. Trillas.

Meixueiro Yeverino, P. (2020). Infancias, desigualdad y participación escolar en tiempos de pandemia. Ichan

Tecolot/. https://ichan.ciesas.edu.mx/infancias-desigualdad-y-participacion-escolar-en-tiempos-de-

pandemia/

Mérida Martínez, Y. y Acuña Gamboa, L. A. (2020). Covid-19, pobreza y educación en Chiapas: análisis a los programas educativos emergentes. Revista Internacional de Educación para la Justicia Social, 9(3e), 61-82.

https://doi.org/10.15366/riejs2020.9.3.004

Muñoz Moreno, J. L. y Lluch Molins, L. (2020). Educación y Covid-19: Colaboración de las familias y tareas

escolares. Revista Internacional de Educación para la Justicia Social, 9(3e).

https://revistas.uam.es/riejs/article/view/12182

Murillo, F. J. (2016) Midiendo la segregación escolar en América Latina. Un análisis metodológico utilizando el TERCE. REICE. Revista Iberoamericana sobre Calidad, Eficacia y Cambio en Educación, 14(4), 33-60.

https://doi.org/10.15366/reice2016.14.4.002

Navarrete Cazales, Z., Manzanilla Granados, H. M. y Ocaña Pérez, L. (2020). Políticas implementadas por el gobierno mexicano frente al Covid-19. El caso de la educación básica. RLEE Nueva Época, /, 143-172.

https://doi.org/10.48102/rlee.2020.50.ESPECIAL.100

Ponce García, G. (2020). Cavilaciones de un profesor en el encierro. La pedagogía infectada y su agónica cuarentena. En J. Navarro Saras (Coord.), Los tiempos educativos en el Covid-19. Historias, vivencias y testimonios desde la escuela distante (pp. 121-129). https://revistaeducarnos.com/wp-

content/uploads/2020/07/covid19-3.pdf

Rodigou Nocetti, M. y Paulín, H. (2013). Coloquios de investigación cualitativa. Desafíos en la investigación como relación social. Universidad Nacional de Córdoba.

Sandín Esteban, M. P. (2003). Investigación cualitativa en educación. Fundamentos y tradiciones. McGraw Hill.

Sanz, I., Sáinz González, J. y Capilla, A. (2020). Efectos de la crisis del coronavirus en la educación. OEl. SEP. (2015). Diagnóstico ampliado del programa de la Reforma Educativa U082. Recuperado el 11/3/2021 de https://www.gob.mx/cms/uploads/attachment/file/50174/Diagno_stico_Ampliado.pdf

Sen, A. y Kliksberg, B. (2007). Primero la gente: Una mirada desde la ética del desarrollo a los principales problemas del mundo globalizado. Ediciones Deusto.

Silva, T. C., Ramos Silva, E. y Montanari, R. (2020). Dificultades de la educación remota en las escuelas rurales del norte de Minas Gerais durante la pandemia de Covid-19. Research, Society and Development, 9(8), http://dx.doi.org/10.33448/rsd-v9i8.6053 Sumner, A., Hoy, C. y Ortiz-Juárez, E. (2020). Estimates of the impact of Covid-19 on global poverty. United Nations University. https://doi.org/10.35188/UNU-WIDER/2020/800-9

Sunkel, G., Trucco, D. y Espejo, A. (2014). La integración de las tecnologías digitales en las escuelas de América Latina y el Caribe. Una mirada multidimensional. CEPAL.

https://repositorio.cepal.org/bitstream/handle/11362/36739/1/S20131120_es.pdf

Tranier, J. (2021a). ¿Presencialidad o "materialidad"? La "cabeza" del (cuerpo) docente como demanda social y control de la enseñanza en contextos de pandemia. Revista Movimiento, 9-11.

https://www.revistamovimiento.com/educacion/presencialidad-o-materialidad-la-cabeza-del-cuerpodocente-como-demanda-social-y-de-control-de-la-ensenanza-en-contextos-de-pandemia/ 
Tranier, J. (2021b). El cuerpo tibio de la docencia. Revista Movimiento, 30, 59-60.

https://www.revistamovimiento.com/educacion/el-cuerpo-tibio-de-la-docencia/

Tranier, J., Bazán, S., Porta, L. y Di Franco, M. G. (2020). Concatenaciones fronterizas: pedagogías,

oportunidades, mundos sensibles y COVID-19. Praxis Educativa, 24(2), 1-18.

https://dx.doi.org/10.19137/praxiseducativa-2020-240203

Trejo Quintana, J. (2020). La falta de acceso y aprovechamiento de los medios y las tecnologías: dos deudas de la educación en México. En H. Casanova Cardiel, Educación y pandemia. Una visión académica (pp. 122129). Instituto de Investigaciones sobre la Universidad y la Educación/UNAM.

Vázquez Soto, M. A., Bonilla Moreno, W. T. y Acosta Rosales, L. Y. (2020). La educación fuera de la escuela en época de pandemia por Covid 19. Experiencias de alumnos y padres de familia. Revista Electrónica Sobre Cuerpos Académicos y Grupos de Investigación, X14), 111-134.

https://www.cagi.org.mx/index.php/CAGl/article/view/213 